\title{
Well-Posedness of the Cauchy Problem for Hyperbolic Equations with Non-Lipschitz Coefficients
}

\author{
Akbar B. Aliev and Gulnara D. Shukurova \\ Baku State University, Academic Zahid Xalilov str., 23, AZ 1148 Baku, Azerbaijan \\ Correspondence should be addressed to Akbar B. Aliev, alievagil@yahoo.com \\ Received 12 March 2009; Accepted 16 May 2009 \\ Recommended by Pavel Sobolevskii \\ We consider hyperbolic equations with anisotropic elliptic part and some non-Lipschitz \\ coefficients. We prove well-posedness of the corresponding Cauchy problem in some functional \\ spaces. These functional spaces have finite smoothness with respect to variables corresponding to \\ regular coefficients and infinite smoothness with respect to variables corresponding to singular \\ coefficients.
}

Copyright (c) 2009 A. B. Aliev and G. D. Shukurova. This is an open access article distributed under the Creative Commons Attribution License, which permits unrestricted use, distribution, and reproduction in any medium, provided the original work is properly cited.

\section{Introduction}

Let us consider the Cauchy problem for a second-order hyperbolic equation:

$$
\begin{gathered}
\ddot{u}-\sum_{i, j=1}^{n} a_{i j}(t) u_{x_{i} x_{j}}+\sum_{j=1}^{n} b_{j}(t) u_{x_{j}}+c(t) u=0, \quad(t, x) \in[0, T] \times R^{n}, \\
u(0, x)=u_{0}(x), \quad u_{t}(0, x)=u_{1}(x), \quad x \in R^{n},
\end{gathered}
$$

where the matrix $\left(a_{i j}(t)\right)$ is real and symmetric for all $t \in(0, T], \ddot{u}=u_{t t}$.

Suppose that (1.1) is strictly hyperbolic, that is, there exists $\lambda_{0}>0$ such that

$$
a(t, \xi) \equiv \sum_{i, j=1}^{n} a_{i j}(t) \frac{\xi_{i} \xi_{j}}{|\xi|^{2}} \geq \lambda_{0}>0
$$

for all $(t, \xi) \in(0, T] \times R^{n} \backslash\{0\}$. 
It is known that if $a(t, \xi)$ satisfies the Lipschitz condition and $b_{j}(t), c(t) \in L_{\infty}(0, T), j=$ $1,2, \ldots, n$, then for any $u_{0} \in H^{s}\left(R^{n}\right), u_{1} \in H^{s-1}\left(R^{n}\right)$ the problem (1.1), (1.2) has a unique solution

$$
u(\cdot) \in C\left([0, T] ; H^{s}\left(R^{n}\right)\right) \cap C^{1}\left([0, T], H^{s-1}\left(R^{n}\right)\right)
$$

where $s \geq 1$ (see [1, Chapter 5] and [2, Chapter 3]) . (see [3]).

If we reject the Lipschitz condition, this result, generally speaking, stops to be valid

In the paper [4] it is proved that if $a(t, \xi) \in L L_{\omega}(0, T)$, that is, if $a(t, \xi)$ satisfies the logarithmic Lipschitz condition:

$$
|a(t+\tau, \xi)-a(t, \xi)| \leq c|\tau| \cdot|\log | \tau|| \cdot \omega(|\tau|)
$$

where $\omega(|\tau|)$ monotonically decreasing tends to zero, and $\log |\tau| \cdot \omega(|\tau|)$ tends to infinity, then there exists $\delta>0$ such that, for all $u_{0} \in H^{s}\left(R^{n}\right), u_{1} \in H^{s-1}\left(R^{n}\right)$ the problem (1.1), (1.2) has a unique solution $u \in C\left([0, T] ; H^{s-\delta}\left(R^{n}\right)\right) \cap C^{1}\left([0, T], H^{s-1-\delta}\left(R^{n}\right)\right)$ (this behavior goes under the name of loss of derivatives).

In the paper [5] it is considered the case when $a_{i, j}(t)=0, i \neq j$, a part of coefficients belongs to the class $L L_{\omega}(0, T)$, and another part of coefficients satisfies the Lipschitz condition. It is proved that the loss of derivatives occurs in those variables $x_{k}$ for which appropriate coefficient $a_{k k}(t)$ belongs to the class $L L_{\omega}(0, T)$.

It is interesting to investigate the Cauchy problem for (1.1), with singular coefficients. Many interesting results have been obtained in this direction. For example, in the paper [6] it is supposed that for each $\xi \in R^{n} \backslash\{0\} a(t, \xi) \in C^{1}(0, T]$ and

$$
t^{q}|\dot{a}(t, \xi)| \leq c, \quad(t, \xi) \in(0, T] \times R^{n} \backslash\{0\},
$$

where $q \geq 1, c>0$. It is proved that if $q=1$, the problem (1.1), (1.2) is well-posed in $C^{\infty}\left(R^{n}\right)$. If $q>1$ and

$$
t^{p}|a(t, \xi)| \leq c, \quad(t, \xi) \in(0, T] \times R^{n} \backslash\{0\}
$$

where $p \in[0,1) \cap[0, q-1)$, then the problem (1.1), (1.2) is well-posed in the Geverey class $\gamma^{(s)}\left(R^{n}\right), s<(q-p) /(q-1)$ (see [6]). If the coefficients $a_{i j}(t)$ satisfy only Holder conditions of order $\alpha<1$ then in [3] it is established that the problem (1.1), (1.2) is $\gamma^{(s)}$ well-posed for all $s<1 /(1-\alpha)$. In this direction see also the results obtained in the papers [6-13].

In this paper we consider the Cauchy problem for a higher-order hyperbolic equation with anisotropic elliptic part:

$$
\begin{gathered}
\ddot{u}+\sum_{k=1}^{n}(-1)^{l_{k}} a_{k}(t) D_{x_{k}}^{2 l_{k}} u+\sum_{|\alpha: l| \leq 1} b_{\alpha}(t) D_{x}^{\alpha} u=0, \quad(t, x) \in[0, T] \times R^{n}, \\
u(0, x)=u_{0}(x), \quad u_{t}(0, x)=u_{1}(x), \quad x \in R^{n},
\end{gathered}
$$

where $l_{k} \in N,\{1,2, \ldots\},, \alpha_{k} \in N \cup\{0\}, k=1,2, \ldots, n,|\alpha: l|=\alpha_{1} / l_{1}+\cdots+\alpha_{n} / l_{n}$. 
Here the coefficients $a_{k}(t)$ satisfy different conditions of type (1.6) and (1.7), so that $q_{k}$ and $p_{k}$ corresponding to different $k$ are different. The smoothness of the solution depending on smoothness on initial data with respect to each variable $x_{k}$ depends not only on $l_{k}$ but also on $q_{k}$ and $p_{k}$.

\section{Statement of the Problem and Results}

We considered the Cauchy problem (1.8). Suppose that $a_{k}(t)$ and $b_{\alpha}(t)$ satisfy the following conditions:

$$
\begin{gathered}
a_{k}(t) \geq a>0, \quad t \in[0, T], k=1,2, \ldots, n, \\
t^{q_{k}}\left|\dot{a}_{k}(t)\right| \leq c, \quad t \in(0, T], \quad k=1,2, \ldots, n, \\
b_{\alpha}(t) \in L_{\infty}(0, T), \quad|\alpha: l| \leq 1 .
\end{gathered}
$$

In order to formulate the basic results we introduce some denotation. Let $H$ be some Hilbert space. By $W_{2}^{\lambda, L}\left(R^{m}, H\right)$ we will denote a functional space with the norm

$$
\|u\|_{W_{2}^{\lambda, L}\left(R^{m}, H\right)}=\left[\int_{R^{m}}\left(1+\sum_{k=1}^{m} \eta_{k}^{2 L_{k}}\right)^{\lambda}\|\widehat{u}(\eta)\|_{H}^{2} d \eta\right]^{1 / 2}
$$

where $L=\left(L_{1}, \ldots, L_{m}\right), L_{j} \in N, j=1,2, \ldots, m, \lambda \geq 0$, and $\widehat{u}(\eta)=F_{x}[u](\eta) ; F_{x}$ is a Fourier transformation with respect to variable $x \in R^{n}$.

For $s \geq 1$ by $\gamma_{\beta}^{s, L}\left(R^{m}, H\right)$ we will denote a functional space with the norm

$$
\|u\|_{\gamma_{\beta}^{s, L}\left(R^{m}, H\right)}=\left[\int \exp \left\{\beta\left|\sum_{k=1}^{m} \eta_{k}^{2}\right|^{1 / s}\right\}\|\widehat{u}(\eta)\|_{H}^{2} d \eta\right]^{1 / 2}
$$

Denote $W_{2}^{\lambda, L}\left(R^{m}, R\right)=W_{2}^{\lambda, L}\left(R^{m}\right), \gamma_{\beta}^{s, L}\left(R^{m}, R\right)=\gamma_{\beta}^{s, L}\left(R^{m}\right)$

$$
C^{\infty}\left(R^{m} ; H\right)=\bigcap_{\lambda \geq 0} W_{2}^{\lambda, L}\left(R^{m} ; H\right), \quad \gamma^{(s)}\left(R^{m} ; H\right)=\bigcap_{\beta \geq 0} r_{\beta}^{(s)}\left(R^{m} ; H\right)
$$

If $L=(1, \ldots, 1)$ then $W_{2}^{\lambda, L}\left(R^{m}, H\right)=H^{\lambda}\left(R^{m} ; H\right), \gamma_{\beta}^{s, L}\left(R^{m}, H\right)=\gamma_{\beta}^{s}\left(R^{m}, H\right)$, and $\gamma_{\beta}^{s, L}\left(R^{m}, R\right)=\gamma_{\beta}^{(s)}$, where $\gamma_{\beta}^{(s)}$ is the Geverey space of order $s$ (see $\left.[12,13]\right)$. If $\lambda \in H$ then $W_{2}^{\lambda, L}\left(R^{m}, H\right)$ is Hilbert-valued anisotropic Sobolev space $W_{2}^{\left(\lambda L_{1}, \ldots, \lambda L_{m}\right)}\left(R^{m} ; H\right)$. For the read valued functions the anisotropic Sobolev spaces are stated in [14]. The basic results led in [14] are also valid for abstract-valued functions. 
We introduce also the following denotation:

$$
\begin{array}{rlrl}
x^{\prime}=\left(x_{1}, \ldots, x_{n_{1}}\right), & x^{\prime \prime} & =\left(x_{n_{1}+1}, \ldots, x_{n}\right), \\
\xi^{\prime}=\left(\xi_{1}, \ldots, \xi_{n_{1}}\right), & \xi^{\prime \prime} & =\left(\xi_{n_{1}+1}, \ldots, \xi_{n}\right), \\
l^{\prime}=\left(l_{1}, \ldots, l_{n_{1}}\right), & l^{\prime \prime}=\left(l_{n_{1}+1}, \ldots, l_{n}\right), \\
|\xi|=\sum_{k=1}^{n} \xi_{k}^{2 l_{k}}, \quad\left|\xi^{\prime}\right|_{l^{\prime}}=\sum_{k=1}^{n_{1}} \xi_{k}^{2 l_{k}}, & \left|\xi^{\prime \prime}\right|_{l^{\prime \prime}}=\sum_{k=n_{1}+1}^{n} \xi_{k}^{2 l_{k}}, \quad n_{2}=n-n_{1} .
\end{array}
$$

The main results are the following theorems.

Theorem 2.1. Let the conditions (2.1)-(2.3) be satisfied, where

$$
\begin{gathered}
q_{k} \in[0,1), \quad \text { for } k=1,2, \ldots, n_{1}, \\
q_{k}=1, \quad \text { for } k=n_{1}+1, \ldots, n .
\end{gathered}
$$

Then for any $\lambda^{\prime} \geq 0, \lambda^{\prime \prime} \geq 0$ the energy estimates

$$
E\left(t, \lambda^{\prime}, \lambda^{\prime \prime}\right) \leq M E\left(0, \lambda^{\prime}, \lambda^{\prime \prime}+\lambda_{0}\right)
$$

hold, where $M$ and $\lambda_{0}$ are some constants indepent of $t \in[0, T]$,

$$
\begin{gathered}
E\left(t, \lambda^{\prime}, \lambda^{\prime \prime}+\lambda\right)=\int_{R^{n}}\left(1+\left|\xi^{\prime}\right|_{l^{\prime}}\right)^{\lambda^{\prime}}\left(1+\left|\xi^{\prime \prime}\right|_{l^{\prime \prime}}\right)^{\lambda^{\prime \prime}+\lambda}\left[|\dot{v}(t, \xi)|^{2}+\left(1+|\xi|_{l}\right)|v(t, \xi)|^{2}\right] d \xi, \\
\curlywedge \geq 0, \quad \dot{v}(t, \xi)=\frac{\partial v(t, \xi)}{\partial t} .
\end{gathered}
$$

Theorem 2.2. Let the conditions (2.1)-(2.3) be satisfied, where

$$
\begin{gathered}
q_{k} \in[0,1), \quad \text { for } k=1,2, \ldots, n_{1}, \\
q_{k}=q>1, \quad \text { for } k=n_{1}+1, \ldots, n .
\end{gathered}
$$

Additionally, let the conditions

$$
t^{p}\left|a_{k}(t)\right| \leq c, \quad t \in[0, T], \quad \text { for } k=n_{1}+1, \ldots, n .
$$

be satisfied, where $p \in[0,1) \cap[0, q-1)$. Then for any $\beta>0, \lambda^{\prime} \geq 0$, and $1 \leq s<(q-p) /(q-1)$ the energy estimates,

$$
\mathcal{\varepsilon}\left(t, \beta, s, \lambda^{\prime}\right) \leq \operatorname{M\varepsilon }\left(0, \beta+\delta, s, \lambda^{\prime}\right)
$$


hold, where $M$ and $\delta$ are some constants independent of $t \in[0, T]$,

$$
\mathcal{E}\left(t, \beta, s, \lambda^{\prime}\right)=\int_{R^{n}} \exp \left\{\beta\left|\xi^{\prime \prime}\right|_{l^{\prime \prime}}^{1 / s}\right\}\left(1+\left|\xi^{\prime}\right|_{l^{\prime}}\right)^{\lambda^{\prime}}\left[|\dot{v}(t, \xi)|^{2}+\left(1+|\xi|_{l}\right)|v(t, \xi)|^{2}\right] d \xi .
$$

Remark 2.3. It is clear by our notation that

$$
\begin{aligned}
E\left(t, \lambda^{\prime}, \lambda^{\prime \prime}\right) \leq & \|\dot{u}(t, \cdot)\|_{W_{2}^{\lambda^{\prime \prime}, l^{\prime \prime}}\left(R_{x^{\prime \prime}}^{n_{2}} ; W_{2}^{\lambda^{\prime}+1, l^{\prime}}\left(R_{x^{\prime}}^{n_{1}}\right)\right)}+\|u(t, \cdot)\|_{W_{2}^{\lambda^{\prime \prime}, l^{\prime \prime}}\left(R_{x^{\prime \prime}}^{n^{\prime \prime}} ; W_{2}^{\lambda^{\prime}+1, l^{\prime}}\left(R_{x^{\prime}}^{n_{1}}\right)\right)} \\
& +\|u(t, \cdot)\|_{W_{2}^{\lambda^{\prime \prime}+1, l^{\prime \prime}}}\left(R_{x^{\prime \prime}}^{n_{2}} ; W_{2}^{\lambda^{\prime},,^{\prime}}\left(R_{x^{\prime}}^{n_{1}}\right)\right) \\
\leq & 2 E\left(\lambda^{\prime}, \lambda^{\prime \prime}, t\right),
\end{aligned}
$$

and we can write

$$
\mathcal{E}\left(t, \beta, s, \lambda^{\prime}\right)=\|u(t, \cdot)\|_{\gamma_{\beta}^{s, l^{\prime \prime}}\left(R_{x^{\prime \prime}}^{n_{2}} ; W_{2}^{\lambda^{\prime}, l^{\prime}}\left(R_{x^{\prime}}^{n_{1}}\right)\right)} \cdot
$$

Remark 2.4. It is possible to replace the conditions $a_{1}(t), \ldots, a_{n_{1}}(t) \in C^{1}(0, T]$ and (2.8) or (2.12) by Lipschitz conditions.

The following theorems are obtained from Theorems 2.1 and 2.2.

Theorem 2.5. Let condition (2.1)-(2.9) be satisfied. Then for any $s \geq 0, u_{0} \in C^{\infty}\left(R_{x^{\prime \prime}}^{n_{2}} ; W_{2}^{s+1, l^{\prime}}\left(R_{x^{\prime}}^{n_{1}}\right)\right)$, $u_{1} \in C^{\infty}\left(R_{x^{\prime \prime}}^{n_{2}} ; W_{2}^{s, l^{\prime}}\left(R_{x^{\prime}}^{n_{1}}\right)\right)$ the problem (1.1), (1.2) admits a unique solution

$$
u \in C\left([0, T] ; C^{\infty}\left(R_{x^{\prime \prime}}^{n_{2}} ; W_{2}^{s+1, l^{\prime}}\left(R_{x^{\prime}}^{n_{1}}\right)\right)\right) \cap C^{1}\left([0, T] ; C^{\infty}\left(R_{x^{\prime \prime}}^{n_{2}} ; W_{2}^{s, l^{\prime}}\left(R_{x^{\prime}}^{n_{1}}\right)\right)\right) .
$$

Theorem 2.6. Let conditions (2.1)-(2.3) and (2.12)-(2.14) be satisfied. Then for any $s^{\prime} \geq 0,1 \leq$ $s^{\prime \prime}<(q-p) /(q-1), u_{0} \in \gamma^{s^{\prime \prime}}\left(R_{x^{\prime \prime}}^{n_{2}} ; W_{2}^{s^{\prime}+1, l^{\prime}}\left(R_{x^{\prime}}^{n_{1}}\right)\right), u_{1} \in \gamma^{s^{\prime \prime}}\left(R_{x^{\prime \prime}}^{n_{2}} ; W_{2}^{s^{\prime}, l^{\prime}}\left(R_{x^{\prime}}^{n_{1}}\right)\right)$ the problem (1.1), (1.2) admits a unique solution

$$
u \in C\left([0, T] ; \gamma^{s^{\prime \prime}}\left(R_{x^{\prime \prime}}^{n_{2}} ; W_{2}^{s^{\prime}+1, l^{\prime}}\left(R_{x^{\prime}}^{n_{1}}\right)\right)\right) \cap C^{1}\left([0, T] ; \gamma^{s^{\prime \prime}}\left(R_{x^{\prime \prime}}^{n_{2}} ; W_{2}^{s^{\prime}, l^{\prime \prime}}\left(R_{x^{\prime}}^{n_{1}}\right)\right)\right) .
$$

In particular it follows from Theorem 2.1 that if the conditions (2.1)-(2.3) are satisfied, then the problem (1.1), (1.2) is well-posed in $C^{\infty}\left(R^{n}\right)$, and if the conditions (2.1)-(2.3) and (2.12)-(2.14) are satisfied then the problem (1.1), (1.2) is well-posed in the Geverey class $\gamma^{(s)}$.

\section{Proof of Theorems}

At first we reduce some auxiliary statements.

We denote $v(t, \xi)=F_{x}[u](t, \xi)$ and define the weighted energetic function in the following way:

$$
\Phi(t)=\Phi\left(t, \xi, \lambda^{\prime}, \lambda^{\prime \prime}, \beta, r\right)=\left[|\dot{v}(t, \xi)|^{2}+\left(1+\left|\xi^{\prime}\right|_{l^{\prime}}+d\left(t, \xi^{\prime \prime}\right)\right)|v(t, \xi)|^{2}\right] \cdot H(t, \xi),
$$


where

$$
\begin{aligned}
& H(t, \xi)=H\left(t, \xi, \lambda^{\prime}, \lambda^{\prime \prime}, \beta, r\right) \\
& =\left(1+\left|\xi^{\prime}\right|_{l^{\prime}}\right)^{\lambda^{\prime}}\left(1+\left|\xi^{\prime \prime}\right|_{l^{\prime \prime}}\right)^{\lambda^{\prime \prime}} \\
& \times \exp \left[-\int_{0}^{t} \alpha\left(\tau, \xi^{\prime \prime}\right) d \tau+\beta\left|\xi^{\prime \prime}\right|_{l^{\prime \prime}}^{(q-1) / r}\right], \quad \lambda^{\prime} \geq 0, \lambda^{\prime \prime} \geq 0, \beta>0, \\
& r= \begin{cases}s(q-1), & \text { for } q>1 \\
1, & \text { for } q=1\end{cases} \\
& d\left(t, \xi^{\prime \prime}\right) \begin{cases}\sum_{k=n_{1}+1}^{n} a_{k}(T) \xi_{k}^{2 l_{k},} & \text { for } T^{r}\left|\xi^{\prime \prime}\right|_{l^{\prime \prime}} \leq 1, \\
\sum_{k=n_{1}+1}^{n} a_{k}\left(\left|\xi^{\prime \prime}\right|_{l^{\prime \prime}}^{-1 / r}\right) \xi_{k}^{2 l_{k},} & \text { for } T^{r}\left|\xi^{\prime \prime}\right|_{l^{\prime \prime}}>1, t^{r}\left|\xi^{\prime \prime}\right|_{l^{\prime \prime}} \leq 1, \\
\sum_{k=n_{1}+1}^{n} a_{k}(t) \xi_{k}^{2 l_{k},} & \text { for } t^{r}\left|\xi^{\prime \prime}\right|_{l^{\prime \prime}}>1,\end{cases} \\
& \alpha\left(t, \xi^{\prime \prime}\right) \begin{cases}\left|d\left(t, \xi^{\prime \prime}\right)-\sum_{k=n_{1}+1}^{n} a_{k}(t) \xi_{k}^{2 l_{k}}\right|, & \text { for } t^{r}\left|\xi^{\prime \prime}\right|_{l^{\prime \prime}} \leq 1, \\
\frac{\left|\sum_{k=n_{1}+1}^{n} \dot{a}_{k}(t) \xi_{k}^{2 l_{k}}\right|}{\sum_{k=n_{1}+1}^{n} a_{k}(t) \xi_{k}^{2 l_{k}},} & \text { for } t^{r}\left|\xi^{\prime \prime}\right|_{l^{\prime \prime}}>1\end{cases}
\end{aligned}
$$

The following auxiliary lemmas are proved similar to the paper [6]. The proofs of the lemmas are in appendix.

Lemma 3.1. If $q_{k}=1, k=n_{1}+1, \ldots, n$, then there exits such $c_{1}>0, c_{2}>0$, that

$$
a\left|\xi^{\prime \prime}\right|_{l^{\prime \prime}} \leq d\left(t, \xi^{\prime \prime}\right) \leq\left[c_{1}+c_{2} \ln \left(1+\left|\xi^{\prime \prime}\right|_{l^{\prime \prime}}\right)\right]\left|\xi^{\prime \prime}\right|_{l^{\prime \prime}}
$$

If $q_{k}>1, k=n_{1}+1, \ldots, n$, then there exits such $c_{1}>0, c_{2}>0$, that

$$
a\left|\xi^{\prime \prime}\right|_{l^{\prime \prime}} \leq d\left(t, \xi^{\prime \prime}\right) \leq\left[c_{1}+c_{2}\left|\xi^{\prime \prime}\right|_{l^{\prime \prime}}^{p / r}\right]\left|\xi^{\prime \prime}\right|_{l^{\prime \prime}}
$$

Lemma 3.2. If $q_{k}=1, k=1,2, \ldots, n_{1}$, then there exits such constant $c_{3}>0, \gamma>0$, that $\int_{0}^{t} \alpha(\tau, \xi) d \tau \leq c_{3}+c_{4} \ln \left(1+\left|\xi^{\prime \prime}\right|_{l^{\prime \prime}}\right)$.

If $q_{k}>1, k=1,2, \ldots, n_{1}$ then there exits such $c_{3}>0, c_{4}>0$, that

$$
\int_{0}^{t} \alpha(\tau, \xi) d \xi \leq c_{3}+c_{4}\left|\xi^{\prime \prime}\right|_{l^{\prime \prime}}^{(q-1) / r}
$$


By the definition of $\Phi(t)=\Phi\left(t, \xi, \lambda^{\prime}, \lambda^{\prime \prime}, \beta, r\right)$ we have

$$
\begin{aligned}
\frac{d \Phi(t)}{d t}= & 2 \operatorname{Re}\left[\ddot{v}(t, \xi) \overline{\dot{v}(t, \xi)}+\left(1+\left|\xi^{\prime}\right|_{\ell^{\prime}}+d\left(t, \xi^{\prime \prime}\right)\right) v(t, \xi) \overline{\dot{v}(t, \xi)}\right] H(t, \xi) \\
& +\dot{d}\left(t, \xi^{\prime \prime}\right)|v(t, \xi)|^{2} H(t, \xi)-\alpha(t, \xi) \Phi(t)
\end{aligned}
$$

On the other hand from (1.8) we have

$$
\begin{gathered}
\ddot{v}(t, \xi)+\sum_{k=1}^{n} a_{k}(t) \xi_{k}^{2 \ell_{k}} v(t, \xi)+\sum_{|\alpha: \ell| \leq 1} b_{\alpha}(t)(i \xi)^{\alpha} v(t, \xi)=0, \\
v(0, \xi)=v_{0}(\xi), \quad \dot{v}(0, \xi)=v_{1}(\xi)
\end{gathered}
$$

where $v_{0}(\xi)=F\left[u_{0}\right](\xi), v_{1}(\xi)=F\left[u_{1}\right](\xi), \ddot{v}(t, \xi)=\partial^{2} v(t, \xi) / \partial t^{2}$

From (3.6) and (3.7) we obtain

$$
\begin{aligned}
\frac{d \Phi(t)}{d t}= & 2 \operatorname{Re}\left[-\sum_{k=1}^{n_{1}} a_{k}(t) \xi_{k}^{2 \ell_{k}}+\left(1+\left|\xi^{\prime}\right|_{\ell^{\prime}}\right)+\left(d\left(t, \xi^{\prime \prime}\right)-\sum_{k=n_{1}+1}^{n} a_{k}(t) \xi_{k}^{2 \ell_{k}}\right)\right] \\
& \times v(t, \xi) \overline{\dot{v}(t, \xi)} H(t, \xi)-2 \operatorname{Re} \sum_{|\alpha: \ell| \leq 1} b_{\alpha}(t)(i \xi)^{\alpha} v(t, \xi) \overline{\dot{v}(t, \xi)} H(t, \xi) \\
& +\dot{d}\left(t, \xi^{\prime \prime}\right)|v(t, \xi)|^{2} H(t, \xi)-\alpha(t, \xi) \Phi(t) .
\end{aligned}
$$

If $t^{r}\left|\xi^{\prime \prime}\right|<1$, then by definition of $d(t, \xi)$ and $\alpha\left(t, \xi^{\prime \prime}\right)$ we have

$$
\begin{aligned}
\frac{d \Phi(t)}{d t}= & 2 \operatorname{Re}\left[-\sum_{k=1}^{n_{1}} a_{k}(t) \xi_{k}^{2 \ell_{k}}+\left(1+\left|\xi^{\prime}\right|_{\ell^{\prime}}\right)+\alpha(t, \xi)\right] v(t, \xi) \overline{\dot{v}(t, \xi)} H(t, \xi) \\
& -2 \operatorname{Re} \sum_{|\alpha: \ell| \leq 1} b_{\alpha}(t)(i \xi)^{\alpha} v(t, \xi) \overline{\dot{v}(t, \xi)} H(t, \xi)-\alpha(t, \xi) \Phi(t) .
\end{aligned}
$$

By our supposition $q_{k}<1$ for $k=1,2, \ldots, n_{1}$. Therefore we can easily see that

$$
a \leq a_{k}(t) \leq a_{T}, \quad k=1,2, \ldots, n_{1}
$$

with some constant $a_{T}>a$. 
Using the Cauchy inequality, definition of $\alpha(t, \xi), H(t, \xi)$, and $\varphi(t)$ we have

$$
\begin{aligned}
& 2 \operatorname{Re} \alpha(t, \xi) v(t, \xi) \overline{\dot{v}(t, \xi)} H(t, \xi)-\alpha(t, \xi) \Phi(t) \leq 0 \\
& 2 \operatorname{Re} \sum_{|\alpha: \ell| \leq 1} b_{\alpha}(t)(i \xi)^{\alpha} v(t, \xi) \overline{\dot{v}(t, \xi)} H(t, \xi) \\
& \quad \leq 2 b_{T} \sum_{|\alpha: l| \leq 1}\left|\xi^{\alpha}\right||v(t, \xi)| \cdot|\dot{v}(t, \xi)| \cdot H(t, \xi) \\
& \quad \leq 2 b_{T} c_{5}\left[\left(1+\sum_{k=1}^{n}\left|\xi_{k}\right|^{2 l_{k}}\right)|v(t, \xi)|^{2}+|\dot{v}(t, \xi)|^{2}\right] H(t, \xi),
\end{aligned}
$$

where $b_{T}=\sup _{|\alpha: l| \leq 1}\left\|b_{\alpha}(t)\right\|_{L_{\infty}(0, T)}, c_{5}=\sup _{\xi \in R^{n}}\left(\sum_{|\alpha: \ell| \leq 1}\left|\xi^{\alpha}\right|\right)^{2} /\left(\sum_{k=1}^{n}\left|\xi_{k}\right|^{2 l_{k}}+1\right)$.

From (3.10)-(3.13) we get that when $t^{r}\left|\xi^{\prime \prime}\right|_{l^{\prime \prime}}<1$, then there exists such a constant $M_{1}>0$, that

$$
\frac{d \Phi(t)}{d t} \leq M_{1} \Phi(t)
$$

If $t^{r}\left|\xi^{\prime \prime}\right|_{l^{\prime \prime}} \geq 1$ then by definition of $d(t, \xi)$ and $\alpha\left(t, \xi^{\prime \prime}\right)$ from (3.9) we have that

$$
\begin{aligned}
\frac{d \Phi(t)}{d t}= & 2 \operatorname{Re}\left[-\sum_{k=1}^{n_{1}} a_{k}(t) \xi_{k}^{2 \ell_{k}}-\sum_{|\alpha: \ell| \leq 1} b_{\alpha}(t)(i \xi)^{\alpha} v(t, \xi) \overline{\dot{v}(t, \xi)}\right] H(t, \xi) \\
& +\sum_{k=n_{1}+1}^{n} \dot{a}_{k}(t) \xi_{k}^{2 \ell_{k}}|v(t, \xi)|^{2} H(t, \xi)-\frac{\left|\sum_{k=n_{1}+1}^{n} \dot{a}_{k}(t) \xi_{k}^{2 \ell_{k}}\right|}{\sum_{k=n_{1}+1}^{n} a_{k}(t) \xi_{k}^{2 \ell_{k}}} \Phi(t) .
\end{aligned}
$$

On the other hand

$$
\begin{aligned}
\sum_{k=n_{1}+1}^{n} \dot{a}_{k}(t) & \xi_{k}^{2 \ell_{k}}|v(t, \xi)|^{2} H(t, \xi)-\frac{\left|\sum_{k=n_{1}+1}^{n} \dot{a}_{k}(t) \xi_{k}^{2 \ell_{k}}\right|}{\sum_{k=n_{1}+1}^{n} a_{k}(t) \xi_{k}^{2 \ell_{k}}} \Phi(t) \\
= & \sum_{k=n_{1}+1}^{n} \dot{a}_{k}(t) \xi_{k}^{2 \ell_{k}}|v(t, \xi)|^{2} H(t, \xi)-\frac{\left|\sum_{k=n_{1}+1}^{n} \dot{a}_{k}(t) \xi_{k}^{2 \ell_{k}}\right|}{\sum_{k=n_{1}+1}^{n} a_{k}(t) \xi_{k}^{2 \ell_{k}}} \\
& \times\left[|\dot{v}(t, \xi)|^{2}+\left(1+\left|\xi^{\prime}\right|_{l^{\prime}}^{2}+\sum_{k=n_{1}+1}^{n} a_{k}(t) \xi_{k}^{2 \ell_{k}}\right)|v(t, \xi)|^{2}\right] H(t, \xi) \leq 0 .
\end{aligned}
$$

From (3.13), (3.15), and (3.16) we again get inequality (3.14).

It follows from (3.14) that

$$
\Phi(t) \leq M \Phi(0), \quad t \in[0, T]
$$

where $M=M_{1} e^{T}$. 
Proof of Theorem 2.1. Let $q_{k}=q=1, k=n_{1}+1, \ldots, n$, then $r=1$. From Lemmas 3.1 and 3.2 we have

$$
\begin{aligned}
\int_{R^{n}} \Phi\left(t, \xi, \lambda^{\prime}, \lambda^{\prime \prime}, 0,1\right) d \xi \leq & \int_{R^{n}}\left(1+\left|\xi^{\prime}\right|_{\ell^{\prime}}\right)^{\lambda^{\prime}}\left(1+\left|\xi^{\prime \prime}\right|_{\ell^{\prime \prime}}\right)^{\lambda^{\prime \prime}} \\
& \times\left[|\dot{v}(t, \xi)|^{2}+\left(1+\left|\xi^{\prime}\right|_{l^{\prime}}^{2}+\left|\xi^{\prime \prime}\right|_{l^{\prime \prime}}\left(c_{1}+c_{2} \ln \left(1+\left|\xi^{\prime \prime}\right|_{l^{\prime \prime}}\right)\right)\right)\right]|v(t, \xi)|^{2} \\
& \times \exp \left(c_{3}+c_{4} \ln \left(1+\left|\xi^{\prime \prime}\right|_{l^{\prime \prime}}\right)\right) d \xi \\
\leq & c_{6} \int_{R^{n}}\left(1+\left|\xi^{\prime}\right|_{\ell^{\prime}}\right)^{\lambda^{\prime}}\left(1+\left|\xi^{\prime \prime}\right|_{\ell^{\prime \prime}}\right)^{\lambda^{\prime \prime}+c_{2}}\left[|\dot{v}(t, \xi)|^{2}+\left(1+|\xi|_{\ell}\right)|v(t, \xi)|^{2}\right] d \xi \\
= & c_{6} E\left(t, \lambda^{\prime}, \lambda^{\prime \prime}+\lambda_{0}\right),
\end{aligned}
$$

where $\lambda_{0}=c_{4}+1, c_{6}=\max \left\{1, c_{1} e^{c_{3}}, c_{2} e^{c_{3}}\right\}$.

Thus,

$$
\int_{R^{n}} \Phi\left(t, \xi, \lambda^{\prime}, \lambda^{\prime \prime}, 0,1\right) d \xi \leq c_{6} E\left(t, \lambda^{\prime}, \lambda^{\prime \prime}+\lambda_{0}\right)
$$

On the other hand from the definition of $\Phi$ and $E$ we have

$$
\int_{R^{n}} \Phi\left(t, \xi, \lambda^{\prime}, \lambda^{\prime \prime}, 0,1\right) d \xi \geq c_{7} E\left(t, \lambda^{\prime}, \lambda^{\prime \prime}\right)
$$

It follows from (3.17)-(3.20) that

$$
E\left(t, \lambda^{\prime}, \lambda^{\prime \prime}\right) \leq c_{8} E\left(0, \lambda^{\prime}, \lambda^{\prime \prime}+d\right)
$$

Proof of Theorem 2.2. Let $q_{k}=q>1, k=n_{1}+1, \ldots, n$, then $r=(q-1)$ s. Taking into account Lemmas 3.1 and 3.2 and Theorem 2.5 we have

$$
\begin{aligned}
\int_{R^{n}} \Phi & \left(t, \xi, \lambda^{\prime}, 0, \beta, r\right) d \xi \\
\leq & \int_{R^{n}}\left(1+\left|\xi^{\prime}\right|_{\ell}\right)^{\lambda^{\prime}}\left[|v(t, \xi)|^{2}+\left(1+\left|\xi^{\prime}\right|_{l^{\prime}}+c_{1}\left|\xi^{\prime \prime}\right|_{l^{\prime}}+c_{2}\left|\xi^{\prime \prime}\right|^{p / 2+1}\right)\right] \\
& \times|v(t, \xi)|^{2} \exp \left(c_{3}+\left.\left(\beta+c_{4}\right)|\xi|\right|^{(q-1) / 2}\right) d \xi
\end{aligned}
$$

Further using the inequality $\eta^{p / 2+1} \leq c_{9} \exp \left(c \eta^{1 / s}\right)$ we obtain

$$
\int_{R^{n}} \Phi\left(t, \xi, \lambda^{\prime}, 0, \beta, r\right) d \xi \leq c_{10} \varepsilon\left(t, \lambda^{\prime}, s, \beta+\delta\right),
$$

where $\delta=c_{4}+c$. 
On the other hand from the definition of $\phi$ and $\varepsilon$ we have

$$
\int_{R^{n}} \Phi\left(t, \xi, \lambda^{\prime}, 0, \beta, r\right) d \xi \geq c_{11} \varepsilon\left(t, \lambda^{\prime}, s, \beta\right) .
$$

From inequalities (3.17), (3.23), and (3.24) it follows that

$$
\varepsilon\left(t, \lambda^{\prime}, s, \beta\right) \leq c_{12} \varepsilon\left(0, \lambda^{\prime}, s, \beta+d\right) \text {. }
$$

Proof of Theorem 2.5. For any $\xi \in R^{n}$ the problem (3.7), (3.8) has a unique solution $v(t, \xi) \in$ $C^{1}[0, T]$ (see $[15$, Chapter I $]$ ).

Let $u_{0} \in C^{\infty}\left(R_{x^{\prime \prime}}^{n_{2}} ; W_{2}^{\lambda^{\prime}+1, l^{\prime}}\left(R_{x^{\prime}}^{n_{1}}\right)\right), u_{1} \in C^{\infty}\left(R_{x^{\prime \prime}}^{n_{2}} ; W_{2}^{\lambda^{\prime}, l^{\prime}}\left(R_{x^{\prime}}^{n_{1}}\right)\right)$, then for any $s \geq 0, \lambda^{\prime} \geq 0$,

$$
E\left(0, \lambda^{\prime}, s+\lambda_{0}\right) \leq c_{s, \lambda^{\prime}}
$$

where $c_{s, \lambda}>0$ is some constant dependent on $s \geq 0$ and $\lambda^{\prime} \geq 0$.

Taking into account Theorem 2.1 it follows from (3.20) that

$$
E\left(t, \lambda^{\prime}, s\right) \leq M c_{\lambda^{\prime}, s,}, \quad t \in[0, T]
$$

that is,

$$
\begin{aligned}
& \|\dot{u}(t, \cdot)\|_{W_{2}^{s, l^{\prime \prime}}\left(R_{x^{\prime \prime}}^{n_{2}} ; W_{2}^{\lambda^{\prime}, l^{\prime}}\left(R_{x^{\prime}}^{n_{1}}\right)\right)}+\|u(t, \cdot)\|_{W_{2}^{s+1, l^{\prime \prime}}\left(R_{x^{\prime \prime}}^{n_{2}} ; W_{2}^{\lambda^{\prime}, l^{\prime}}\left(R_{x^{\prime}}^{n_{1}}\right)\right)} \\
& \quad+\|u(t, \cdot)\|_{W_{2}^{s, l^{\prime \prime}}\left(R_{x^{\prime \prime}}^{n_{2}} ; W_{2}^{\lambda^{\prime}+1, l^{\prime}}\left(R_{x^{\prime}}^{n_{1}}\right)\right)} \leq M c_{\lambda^{\prime}, s,}, \quad t \in[0, T] .
\end{aligned}
$$

It follows from (3.28) that

$$
\begin{aligned}
& u \in C\left([0, T] ; C^{\infty}\left(R_{x^{\prime \prime}}^{n_{2}} ; W_{2}^{\lambda^{\prime}+1, l^{\prime}}\left(R_{x^{\prime}}^{n_{1}}\right)\right)\right), \\
& \dot{u} \in C\left([0, T] ; C^{\infty}\left(R_{x^{\prime \prime}}^{n_{2}} ; W_{2}^{\lambda^{\prime}, l^{\prime}}\left(R_{x^{\prime}}^{n_{1}}\right)\right)\right) .
\end{aligned}
$$

By the expression of $u(t, x)$ it follows that the function $u(t, x)$ is the solution of problem The uniqueness of the solution is proved by standard method.

The proof of Theorem 2.6 is carried out in the similar way. 


\section{Appendices}

\section{A. Proof of Lemmas}

Proof of Lemma 3.1. Let $q_{k}=1, k=n_{1}+1, \ldots, n$. Then from (2.2) we have

$$
\begin{aligned}
a_{k}(t) & \leq a_{k}(T)+\left|a_{k}(t)-a_{k}(T)\right| \\
& \leq a_{k}(T)+\int_{t}^{T}\left|\dot{a}_{k}(s)\right| d s \\
& \leq a_{k}(T)+c \ln \frac{T}{t} \\
& \leq c_{1}+c_{2} \ln \left(1+\frac{1}{t}\right) .
\end{aligned}
$$

It follows from (2.1) and (2.14) that

$$
a\left|\xi^{\prime \prime}\right|_{l^{\prime \prime}} \leq d\left(t, \xi^{\prime \prime}\right)
$$

By definition of $d\left(t, \xi^{\prime \prime}\right)$ for $T\left|\xi^{\prime \prime}\right|_{l^{\prime \prime}} \leq 1$ we have

$$
d\left(t, \xi^{\prime \prime}\right)=\sum_{k=n_{1}}^{n} a_{k}(T) \xi_{k}^{2 l_{k}} \leq c_{1}\left|\xi^{\prime \prime}\right|_{l^{\prime \prime}}
$$

If $T\left|\xi^{\prime \prime}\right|_{l^{\prime \prime}}>1$, and $t\left|\xi^{\prime \prime}\right|_{l^{\prime \prime}}<1$, then from (A.1) we have

$$
\begin{aligned}
d\left(t, \xi^{\prime \prime}\right) & =\sum_{k=n_{1}}^{n} a_{k}\left(\left|\xi^{\prime \prime}\right|_{l^{\prime \prime}}^{-1}\right) \xi_{k}^{2 l_{k}} \\
& \leq\left[c_{1}+c_{2} \ln \left(1+\frac{1}{\left|\xi^{\prime \prime}\right|_{l^{\prime \prime}}^{-1}}\right)\right] \sum_{k=n_{1}}^{n} \xi_{k}^{2 l_{k}} \\
& =\left(c_{1}+c_{2} \ln \left(1+\left|\xi^{\prime \prime}\right|_{l^{\prime \prime}}\right)\right)\left|\xi^{\prime \prime}\right|_{l^{\prime \prime}} .
\end{aligned}
$$

If $t\left|\xi^{\prime \prime}\right|_{l^{\prime \prime}}>1$, then using (A.1) we get

$$
\begin{aligned}
d\left(t, \xi^{\prime \prime}\right) & =\sum_{k=n_{1}+1}^{n} a_{k}(t) \xi_{k}^{2 l_{k}} \\
& \leq\left[c_{1}+c_{2} \ln \left(1+\frac{1}{t}\right)\right]\left|\xi^{\prime \prime}\right|_{l^{\prime \prime}} \\
& \leq\left[c_{1}+c_{2} \ln \left(1+\left|\xi^{\prime \prime}\right|_{l^{\prime \prime}}\right)\right]\left|\xi^{\prime \prime}\right|_{l^{\prime \prime}} .
\end{aligned}
$$

Consequently if $q=1$, the statement of the lemma follows from (A.2)-(A.5). 
Let $q_{k}>1, k=n_{1}+1, \ldots, n$. By definition of $d\left(t, \xi^{\prime \prime}\right)$ for $T^{r}\left|\xi^{\prime \prime}\right|_{l^{\prime \prime}}<1$ we have

$$
d\left(t, \xi^{\prime \prime}\right) \leq c_{1}\left|\xi^{\prime \prime}\right|_{l^{\prime \prime}}
$$

If $T^{r}\left|\xi^{\prime \prime}\right|_{l^{\prime \prime}}>1$ and $t^{r}\left|\xi^{\prime \prime}\right|<1$ then

$$
\begin{aligned}
d\left(t, \xi^{\prime \prime}\right) & =\sum_{k=n_{1}+1}^{n} a_{k}\left(\left|\xi^{\prime \prime}\right|_{l^{\prime \prime}}^{-1 / r}\right) \xi_{k}^{2 l_{k}} \\
& \leq \sum_{k=n_{1}+1}^{n} \frac{M}{\left(\left|\xi^{\prime \prime}\right|_{l^{\prime \prime}}^{-1 / r}\right)^{p}} \xi_{k}^{2 l_{k}} \\
& =M\left|\xi^{\prime \prime}\right|_{l^{\prime \prime}}^{1+\mathrm{p} / r} .
\end{aligned}
$$

If $t^{r}\left|\xi^{\prime \prime}\right|>1$ then

$$
\begin{aligned}
d\left(t, \xi^{\prime \prime}\right) & =\sum_{k=n_{1}+1}^{n} a_{k}(t) \xi_{k}^{2 l_{k}} \\
& \leq \sum_{k=n_{1}+1}^{n} \frac{M}{t^{p}} \xi_{k}^{2 l_{k}} \\
& =M\left|\xi^{\prime \prime}\right|_{l^{\prime \prime}} \cdot\left|\xi^{\prime \prime}\right|^{p / r} \\
& =M\left|\xi^{\prime \prime}\right|^{1+p / r} .
\end{aligned}
$$

Thus if $q_{k}>1, k=n_{1}+1, \ldots, n$ then the statement of the lemma follows from (A.2), (A.6), and (A.8).

The lemma is proved.

Proof of Lemma 3.2. At first we consider the case when $q_{k}=1, k=n_{1}+1, \ldots, n$. If $T\left|\xi^{\prime \prime}\right|_{\ell^{\prime \prime}} \leq 1$, then

$$
\begin{aligned}
\int_{0}^{t} \alpha\left(\tau, \xi^{\prime \prime}\right) d \tau & \leq \int_{0}^{T} \alpha\left(\tau, \xi^{\prime \prime}\right) d \tau \\
& \leq \int_{0}^{T}\left|\sum_{k=n_{1}}^{n} a_{k}(T) \xi_{k}^{2 \ell_{k}}-\sum_{k=n_{1}}^{n} a_{k}(\tau) \xi_{k}^{2 \ell_{k}}\right| d \tau \\
& \leq \sum_{k=n_{1}}^{n} \xi_{k}^{2 \ell_{k}} \int_{0}^{T}\left|a_{k}(T)-a_{k}(\tau)\right| d \tau \\
& \leq T \cdot \max _{k=n_{1}+1, \ldots, n} a_{k}(T)\left|\xi^{\prime \prime}\right|_{\ell^{\prime \prime}}+\left|\xi_{k}^{\prime \prime}\right|_{\ell^{\prime \prime}} \int_{0}^{T} a_{k}(\tau) d \tau \\
& \leq a_{T}
\end{aligned}
$$

where $a_{T}=\max _{k=n_{1}+1, \ldots, n} a_{k}(\tau)+(1 / T) \max _{k=n_{1}+1, \ldots, n} \int_{0}^{T} a_{k}(\tau) d \tau<+\infty$. 
If $T\left|\xi^{\prime \prime}\right|>1$, then

$$
\begin{aligned}
& \int_{0}^{t} \alpha\left(\tau, \xi^{\prime \prime}\right) d s \leq \int_{0}^{\left|\xi^{\prime \prime}\right|_{l^{\prime \prime}}^{-1}} \alpha\left(s, \xi^{\prime \prime}\right) d \tau+\int_{\left|\xi^{\prime \prime}\right|_{\ell^{\prime \prime}}^{-1}}^{T} \alpha\left(s, \xi^{\prime \prime}\right) d s \\
& \leq \int_{0}^{\left|\xi^{\prime \prime}\right| l^{\prime \prime}}\left|d\left(\tau, \xi^{\prime \prime}\right)-\sum_{k=n_{1}}^{n} a_{k}(\tau) \xi_{k}^{2 l_{k}}\right| d \tau+\int_{\left|\xi^{\prime \prime}\right| \ell^{\prime \prime}}^{T} \frac{\left|\sum_{k=n_{1}}^{n} \dot{a}_{k}(\tau) \xi_{k}^{2 \ell_{k}}\right|}{\sum_{k=n_{1}}^{n} a_{k}(\tau) \xi_{k}^{2 \ell_{k}}} d \tau \\
& \leq \int_{0}^{\left|\xi^{\prime \prime}\right| l^{\prime \prime}} d\left(\tau, \xi^{\prime \prime}\right) d \tau+\sum_{k=n_{1}}^{n} \xi_{k}^{2 l_{k}} \cdot \int_{0}^{\left|\xi^{\prime \prime}\right| l^{\prime \prime}} \alpha_{k}(\tau) d \tau+\frac{c}{a} \sum_{k=n_{1}}^{n} \xi_{k}^{2 \ell_{k}} \int_{\left|\xi^{\prime \prime}\right|}^{T} \frac{d \tau}{\tau} \\
& \leq \int_{0}^{\left.\left|\xi^{\prime \prime}\right|\right|_{\ell^{\prime \prime}} ^{-1}}\left[c_{1}+c_{2} \ln \left(1+\left|\xi^{\prime \prime}\right|_{\ell^{\prime \prime}}\right)\right]\left|\xi^{\prime \prime}\right|_{\ell^{\prime \prime}} d \tau \\
& +\sum_{k=n_{1}}^{n} \xi_{k}^{2 \ell_{k}} \cdot c \int_{0}^{\left|\xi^{\prime \prime}\right| \ell^{\prime \prime}} \ln \frac{T}{\tau} d \tau+\frac{c}{a} \sum_{k=n_{1}}^{n} \int_{\left.\left|\xi^{\prime \prime}\right|\right|_{\ell^{\prime \prime}}}^{T} \frac{d \tau}{\tau} \\
& =c_{1}+c_{2} \ln \left(1+\left|\xi^{\prime \prime}\right|_{\ell^{\prime \prime}}^{-1}\right)+c\left|\xi^{\prime \prime}\right|_{\ell^{\prime \prime}} \cdot \int_{0}^{\left.\left|\xi^{\prime \prime}\right|\right|_{\ell^{\prime \prime}} ^{-1}} \ln \frac{T}{\tau} d \tau+\frac{c}{a} \int_{\left|\xi^{\prime \prime}\right|}^{T} \frac{d \tau}{\ell_{\ell^{\prime \prime}}} \frac{d}{\tau} \\
& \leq c_{3}+c_{4} \ln \left(1+\left|\xi^{\prime \prime}\right|_{\ell^{\prime \prime}}\right) .
\end{aligned}
$$

Now let us consider the case $q_{k}>1, k=n_{1}+1, \ldots, n$. In this case $r=(q-1) s$. If $T^{r}\left|\xi^{\prime \prime}\right|_{\ell^{\prime \prime}} \leq 1$, then

$$
\begin{aligned}
\int_{0}^{t} \alpha\left(\tau, \xi^{\prime \prime}\right) d \tau & \leq \int_{0}^{T} \alpha\left(\tau, \xi^{\prime \prime}\right) d \tau \\
& \leq \sum_{k=n_{1}+1}^{n} \int_{0}^{T}\left|a_{k}(T)-a_{k}(\tau)\right| \xi_{k}^{2 \ell_{k}} d \tau \\
& \leq \max _{k=n_{1}+1, \ldots, n} a_{k}(T) T\left|\xi^{\prime \prime}\right|_{\ell^{\prime \prime}}+\int_{0}^{T} c \tau^{-p} d \tau\left|\xi^{\prime \prime}\right|_{\ell^{\prime \prime}} \\
& \leq a_{T} \cdot T^{1-r}+c \cdot \frac{1}{1-p} T^{1-p}\left|\xi^{\prime \prime}\right|_{\ell^{\prime \prime}} \leq a_{T} T^{1-r}+\frac{c}{1-p} T^{1-p-r} .
\end{aligned}
$$


If $T^{r}\left|\xi^{\prime \prime \prime}\right|_{\ell^{\prime \prime}}>1$, then

$$
\begin{aligned}
& \int_{0}^{t} \alpha\left(\tau, \xi^{\prime \prime}\right) d \tau \leq \int_{0}^{\left|\xi^{\prime \prime}\right|^{-1 / r}} \alpha(\tau, \xi) d \tau+\int_{\left|\xi^{\prime \prime}\right|_{\ell^{\prime \prime}}^{-1 / r}}^{T} \alpha\left(\tau, \xi^{\prime \prime}\right) d \tau \\
& \leq \int_{0}^{\left.\xi^{\prime \prime}\right|_{\ell^{\prime \prime}} ^{-1 / \mathrm{r}}}\left|d(\tau, \xi)-\sum_{k=n_{1}+1}^{n} a_{k}(\tau) \xi_{k}^{2 l_{k}}\right| d \tau+\int_{\left|\xi^{\prime \prime}\right|_{\ell^{\prime \prime}}^{-1 / \mathrm{r}}}^{T} \alpha\left(\tau, \xi^{\prime \prime}\right) d \tau \\
& \leq \sum_{k=n_{1}+1}^{n} a_{k}\left(\left|\xi^{\prime \prime}\right|_{\ell^{\prime \prime}}^{-1 / \mathrm{r}}\right) \xi_{k}^{2 \ell_{k}} \int_{0}^{\left|\xi^{\prime \prime}\right|-1 / \mathrm{r}} d \tau+\sum_{l^{\prime \prime}}^{n} \xi_{k}^{2 \ell_{k}} \int_{0}^{\left|\xi^{\prime \prime}\right|_{\ell^{\prime \prime}}^{-1 / \mathrm{r}}} a_{k}(\tau) d \tau \\
& +\int_{\left.\left|\xi^{\prime \prime}\right|\right|_{\ell^{\prime \prime}} ^{-1 / r}}^{T} \frac{\left|\sum_{k=n_{1}+1}^{n} \dot{a}_{k}(\tau) \xi_{k}^{2 \ell_{k}}\right|}{\sum_{k=n_{1}+1}^{n} a_{k}(\tau) \xi_{k}^{2 \ell_{k}}} d \tau \\
& \leq \frac{c}{\left(\left|\xi^{\prime \prime}\right|_{\ell^{\prime \prime}}^{-1 / \mathrm{r}}\right)^{p}} \cdot\left|\xi^{\prime \prime}\right|_{\ell^{\prime \prime}} \cdot \int_{0}^{\left|\xi^{\prime \prime}\right|_{\ell^{\prime \prime}}^{-1 / \mathrm{r}}} d \tau+c\left|\xi^{\prime \prime}\right|_{\ell^{\prime \prime}} \cdot \int_{0}^{\left|\xi^{\prime \prime}\right|^{-1 / r}} \frac{d \tau}{\tau^{p}} d \tau+\frac{c}{a} \int_{\left|\xi^{\prime \prime}\right|^{-1 / r}}^{T} \frac{d \tau}{\tau^{q}} \\
& \leq c\left|\xi^{\prime \prime}\right|_{\ell^{\prime \prime}}^{p / r+1} \cdot\left|\xi^{\prime \prime}\right|^{-1 / r}+c\left|\xi^{\prime \prime}\right|_{\ell^{\prime \prime}} \cdot \frac{1}{1-p}\left(\left|\xi^{\prime \prime}\right|^{-1 / r}\right)^{1-p} \\
& +\frac{c}{a} \frac{1}{1-q}\left(T^{1-q}-\left(\left|\xi^{\prime \prime}\right|^{-1 / r}\right)^{1-q}\right) \\
& <c\left|\xi^{\prime \prime}\right|_{\ell^{\prime \prime}}^{1-((1-p) / r)}+\frac{c}{1-p}\left|\xi^{\prime \prime}\right|_{\ell^{\prime \prime}}^{1-((1-p) / r)}+\frac{c}{a(q-1)}\left|\xi^{\prime \prime}\right|^{(q-1) / r} .
\end{aligned}
$$

As $r=(q-1) s$, and $s<(q-p) /(q-1)$, it follows that $1-(1-p) / s<1 / s$ and $(q-1) / r=1 / s$. Then according to the Young inequality there exists such $\delta>0$ that

$$
\left|\xi^{\prime \prime}\right|^{1-((1-p) / r)} \leq c_{1} \delta+\delta_{1}\left|\xi^{\prime \prime}\right|^{1 / s}
$$

Thus, by (A.9)-(A.13) the following inequality is valid:

$$
\int_{0}^{t} \alpha\left(\tau, \xi^{\prime \prime}\right) d \tau \leq \delta|\xi|^{1 / s}+c_{\delta}
$$

where $\delta=\delta_{1} a(2+p) /(1-p)+(c / a(q-1)) c_{\delta}=c_{1 \delta} c(2+\delta) /(1-p)$. 


\section{B. Example}

Let us consider the Cauchy problem in $[0, T) \times R^{2}$ :

$$
\begin{gathered}
u_{t t}-\left(1+t^{2}\right) u_{x x}-\left(1+\sqrt[3]{t^{2}}\right) u_{y y}=0 \\
u(0, x, y)=\varphi_{1}(x) \psi_{1}(y) \\
u_{t}(o, x, y)=\varphi_{2}(x) \psi_{2}(y)
\end{gathered}
$$

where $\varphi_{1}(x), \varphi_{2}(x) \in C^{\infty}(R)=\cap_{s \geq 0} W_{2}^{s}(R), \psi_{1}(y) \in W_{2}^{2}(R), \psi_{2}(y) \in W_{2}^{1}(R), u=u(t, x, y)$.

It follows from Theorem 2.5 that the problem (B.1) has a unique solution

$$
u \in C\left([0, T] ; C^{\infty}\left(R ; W_{2}^{2}(R)\right)\right) \cap C^{1}\left([0, T] ; C^{\infty}\left(R ; W_{2}^{1}(R)\right)\right)
$$

\section{References}

[1] J.-L. Lions and E. Magenes, Problems aux Limites Non-Homogénes et Applications, Dunod, Paris, France, 1968.

[2] L. Hörmander, Linear Partial Differential operators, Springer, Berlin, Germany, 1963.

[3] F. Colombini and E. S. De Giorge, "Existence et uniqute des solutions des equations hyperboluques du second orde a coefficients no dependant," Comptes Rendus de l'Académie des Sciences, vol. 286, pp. 1045-1051, 1978.

[4] M. Cicognani and F. Colombini, "Modulus of continuity of the coefficients and loss of derivatives in the strictly hyperbolic Cauchy problem," Journal of Differential Equations, vol. 221, no. 1, pp. 143-157, 2006.

[5] A. B. Aliev and G. D. Shukurova, "The Cauchy problem for hyperbolic equations with nonsmooth coefficients," in Functional-Differential Equations and Their Applications, Materials of the III International Scientific Conference, pp. 23-30, Mahachkala, Russia, September 2007.

[6] F. Colombini, D. Del Santo, and T. Kinoshita, "Well-posedness of the cauchy problem for hyperbolic equations with non Lipschitz coefficients," Annali della Scuola Normale Superiore di Pisa, vol. 1, pp. 327-358, 2002.

[7] F. Colombini and N. Lerner, "Hyperbolic operators with non-Lipschitz coefficients," Duke Mathematical Journal, vol. 77, no. 3, pp. 657-698, 1995.

[8] M. Reissig, "About strictly hyperbolic operators with non-regular coefficients," Pliska Studia Mathematica Bulgarica, vol. 15, pp. 105-130, 2003.

[9] F. Hirosawa, "Loss of regularity for second order hyperbolic equations with singular coefficients," Osaka Journal of Mathematics, vol. 42, no. 4, pp. 767-790, 2005.

[10] F. Hirosawa and M. Reissig, "Well-posedness in Sobolev spaces for second-order strictly hyperbolic equations with nondifferentiable oscillating coefficients," Annals of Global Analysis and Geometry, vol. 25, no. 2, pp. 99-119, 2004.

[11] F. Hirosawa and M. Reissig, "About the optimality of oscillations in non-Lipschitz coefficients for strictly hyperbolic equations," Annali della Scuola Normale Superiore di Pisa, vol. 3, no. 3, pp. 589-608, 2004.

[12] E. Janelli, "Regularly hyperbolic equations without local solvability," Annales Scientifiques de l'École Normale Supérieure, vol. 22, pp. 109-125, 1989.

[13] T. Nishitani, "Sur los equations hyperboliques a coefficients Hölderiens ent et de classe de Gevrey en x," Bulletin des Sciences Mathématiques, vol. 107, no. 2, pp. 113-138, 1983.

[14] O. V. Besov, V. P. Il'in, and S. M. Nikolskii, Integral Representations of Functions and Embedding Theorem, V.H. Wilson and Sons, Washington, DC, USA, 1978.

[15] E. A. Coddington and N. Levinson, Theory of Ordinary Differential Equations, McGraw-Hill, New York, NY, USA, 1955. 


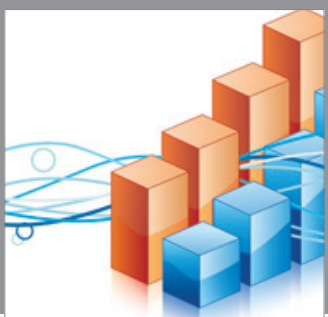

Advances in

Operations Research

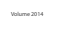

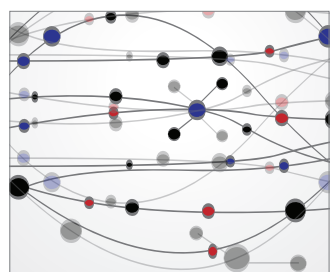

\section{The Scientific} World Journal
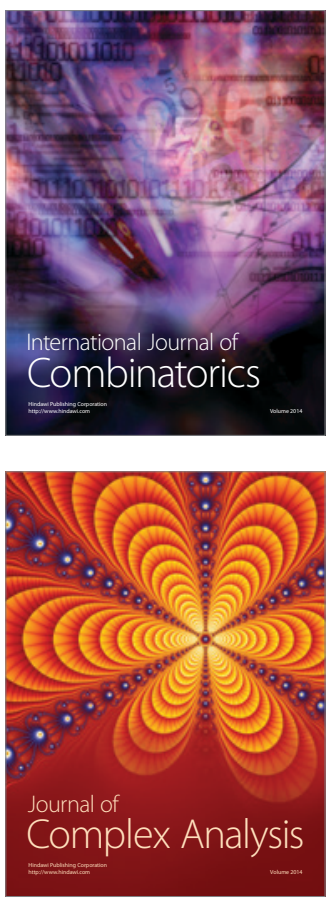

International Journal of

Mathematics and

Mathematical

Sciences
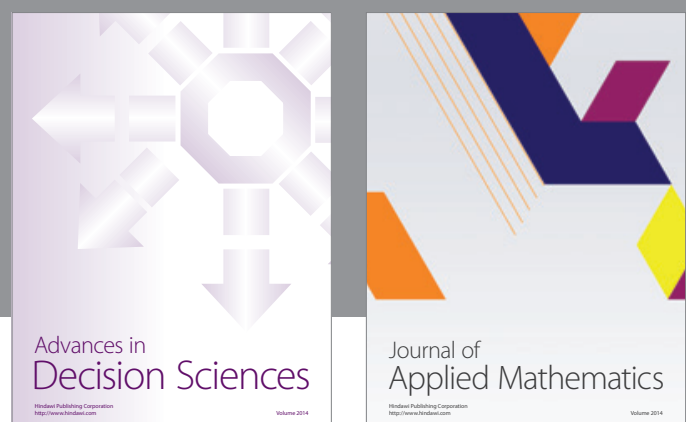

Journal of

Applied Mathematics
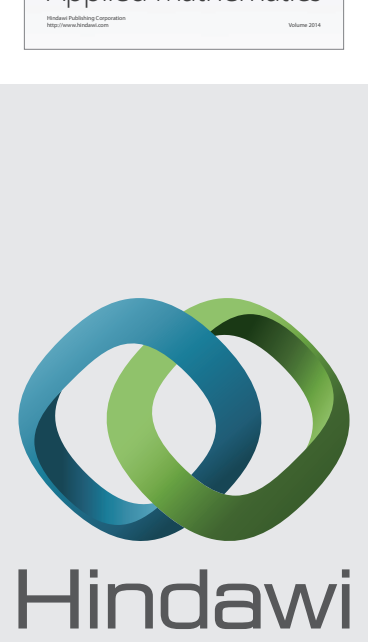

Submit your manuscripts at http://www.hindawi.com
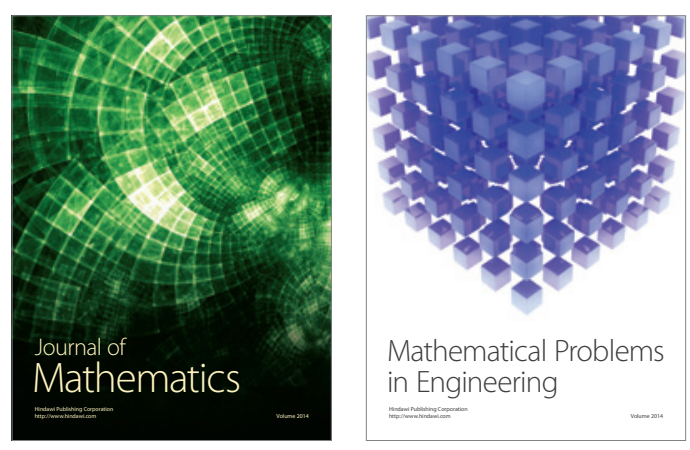

Mathematical Problems in Engineering
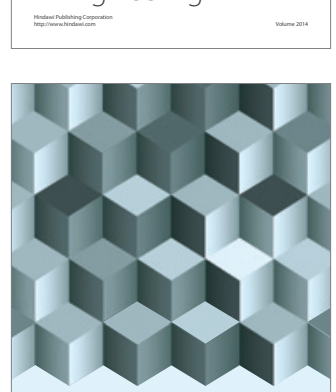

Journal of

Function Spaces
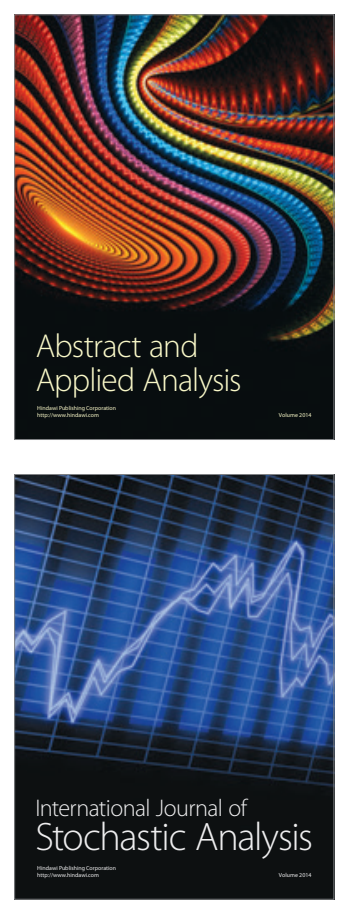

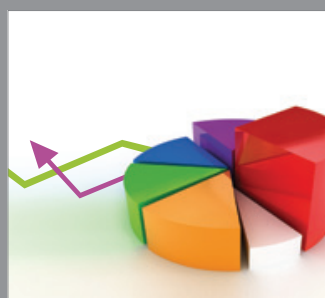

ournal of

Probability and Statistics

Promensencen
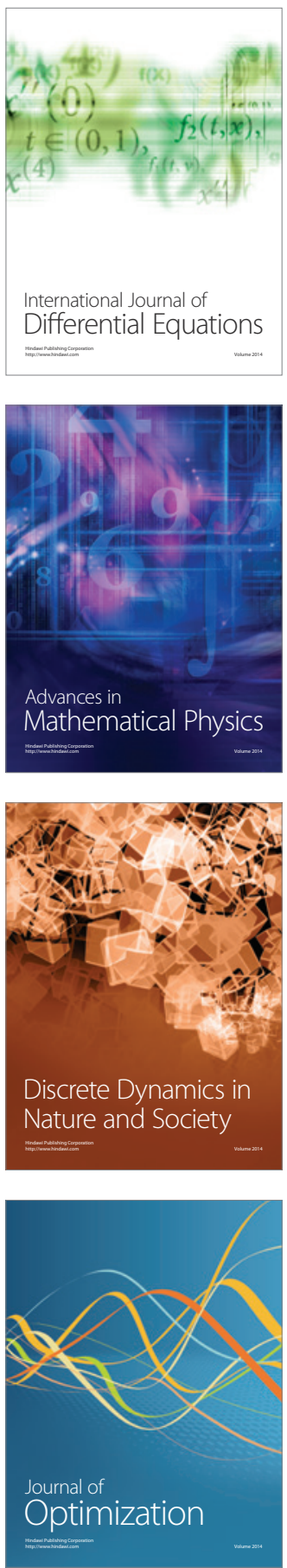